\title{
ON NONLINEAR INTERPOLATION
}

\author{
T. KAPPELER AND P. TOPALOV
}

(Communicated by Yingfei Yi)

\begin{abstract}
In a case study on asymptotics of spectral quantities of Schrödinger operators in fractional Sobolev spaces on the circle we show how a nonlinear version of the Riesz-Thorin theorem on the interpolation of linear operators can be applied.
\end{abstract}

\section{INTRODUCTION}

The aim of this paper is to derive asymptotics of spectral quantities of Schrödinger operators on the circle for potentials in fractional Sobolev spaces by a suitable theorem on nonlinear interpolation which we now state in the set-up used for our applications.

For any $0 \leq s<\infty$ denote by $H_{\mathbb{C}}^{s} \equiv H^{s}(\mathbb{T}, \mathbb{C})$ the complex Sobolev space of order $s$ of the $1 d$ torus $\mathbb{T}=\mathbb{R} / \mathbb{Z}$. Its elements can be represented by a Fourier series $\varphi(x)=\sum_{k \in \mathbb{Z}} \hat{\varphi}(k) e^{2 \pi i k x}$, where $\hat{\varphi}(k):=\int_{0}^{1} \varphi(x) e^{-2 \pi i k x} d x \in \mathbb{C}$, and

$$
\|\varphi\|_{s}:=\left(\sum_{k \in \mathbb{Z}}(1+|k|)^{2 s}|\hat{\varphi}(k)|^{2}\right)^{1 / 2}<\infty .
$$

Note that $H_{\mathbb{C}}^{s^{\prime}} \hookrightarrow H_{\mathbb{C}}^{s}$ for any $0 \leq s \leq s^{\prime}$. Denote by $B_{\mathbb{C}}^{s}(R) \subseteq H_{\mathbb{C}}^{s}$ the open ball of radius $R$, centered at 0 . Furthermore, for any $1 \leq p<\infty$ and $t \in \mathbb{R}$ denote by $\ell_{\mathbb{C}}^{p, t} \equiv \ell^{p, t}(\mathbb{Z}, \mathbb{C})$ the complex sequence space

$$
\ell_{\mathbb{C}}^{p, t}=\left\{\xi=\left(\xi_{j}\right)_{j \in \mathbb{Z}} \subseteq \mathbb{C} \mid\|\xi\|_{p, t}<\infty\right\}
$$

where

$$
\|\xi\|_{p, t}:=\left(\sum_{j \in \mathbb{Z}}\left((1+|j|)^{t}\left|\xi_{j}\right|\right)^{p}\right)^{1 / p} .
$$

Note that $\ell_{\mathbb{C}}^{p, t^{\prime}} \hookrightarrow \ell_{\mathbb{C}}^{p, t}$ for any $0 \leq t \leq t^{\prime}$. Finally, for $b>a \geq 0$ and $\alpha \geq 0, \beta>0$, assume that $F: B_{\mathbb{C}}^{a}(R) \rightarrow \ell_{\mathbb{C}}^{p, \alpha+\beta a}$ is an analytic map, bounded by $M_{a}>0$, so that for some $M_{b}>0$,

$$
\sup _{\varphi \in B_{\mathbb{C}}^{b}(R)}\|F(\varphi)\|_{p, \alpha+\beta b} \leq M_{b}
$$

Received by the editors July 15, 2013.

2010 Mathematics Subject Classification. Primary 46B70, 46B45, 47J35.

The first author was supported in part by the Swiss National Science Foundation.

The second author was supported in part by the NSF grant DMS-0901443. 
By the characterization of analytic maps with sequence spaces as their range (cf. e.g. [5, Appendix A]) and the analyticity of $F$ it follows from (1.1) that $\left.F\right|_{B_{\mathbb{C}}^{b}(R)}: B_{\mathbb{C}}^{b}(R) \rightarrow \ell_{\mathbb{C}}^{p, \alpha+\beta b}$ is analytic as well. Hence, equivalently, we are given the following commutative diagram:

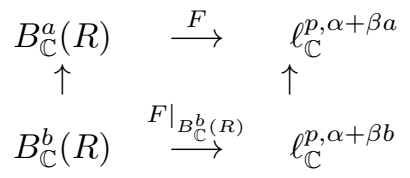

where the horizontal maps $F$ and $\left.F\right|_{B_{\mathbb{C}}^{b}(R)}$ are analytic,

$$
\sup _{\varphi \in B_{\mathbb{C}}^{a}(R)}\|F(\varphi)\|_{p, \alpha+\beta a} \leq M_{a}, \quad \sup _{\varphi \in B_{\mathbb{C}}^{b}(R)}\|F(\varphi)\|_{p, \alpha+\beta b} \leq M_{b},
$$

and the vertical arrows denote the standard inclusions of the corresponding spaces.

The following theorem is an instance of an extension of the Riesz-Thorin theorem (cf. e.g. [1]) to nonlinear maps and is inspired by a special case treated in 6. Appendix 2].

Theorem 1.1. Under the assumptions stated above, $F$ interpolates between $B_{\mathbb{C}}^{a}(R)$ and $B_{\mathbb{C}}^{b}(R)$. More precisely, for any $s=(1-\lambda) a+\lambda b$ with $0 \leq \lambda \leq 1, F\left(B_{\mathbb{C}}^{s}(R)\right) \subseteq$ $\ell_{\mathbb{C}}^{p, \alpha+\beta s}$ and $\left.F\right|_{B_{\mathbb{C}}^{s}(R)}: B_{\mathbb{C}}^{s}(R) \rightarrow \ell_{\mathbb{C}}^{p, \alpha+\beta s}$ is an analytic map, bounded by $M_{a}^{1-\lambda} M_{b}^{\lambda}$.

The proof of Theorem 1.1. presented in Section 2, uses in a crucial way the assumption that $F$ is analytic on a ball in a complex (Hilbert) space. The proof does not apply to nonlinear maps

$$
F: B_{\mathbb{C}}^{s}(R) \cap H^{s}(\mathbb{T}, \mathbb{R}) \rightarrow \ell^{p, \alpha+\beta s}(\mathbb{Z}, \mathbb{R})
$$

which are merely real analytic. In the sequel we would like to discuss a type of problem where nevertheless Theorem 1.1 can be applied to real analytic nonlinear maps. For $q$ in $L_{0}^{2}(\mathbb{T}, \mathbb{R})=\left\{q \in L^{2}(\mathbb{T}, \mathbb{R}) \mid \int_{0}^{1} q(x) d x=0\right\}$ let $L(q)$ be the Schrödinger operator $L(q):=-d_{x}^{2}+q$. The Dirichlet spectrum of $L(q)$, considered on the interval $[0,1]$, is real and consists of simple eigenvalues. We list them in increasing order $\mu_{1}<\mu_{2}<\ldots$. Furthermore denote by $M(x, \lambda)$ the fundamental solution of $L(q)$, i.e. the $2 \times 2$ matrix valued function satisfying $L(q) M=\lambda M, \lambda \in \mathbb{C}$, and $M(0, \lambda)=I d_{2 \times 2}$,

$$
M(x, \lambda)=\left(\begin{array}{ll}
y_{1}(x, \lambda) & y_{2}(x, \lambda) \\
y_{1}^{\prime}(x, \lambda) & y_{2}^{\prime}(x, \lambda)
\end{array}\right)
$$

When evaluated at $\lambda=\mu_{n}$, the Floquet matrix $M(1, \lambda)$ is lower triangular, hence its eigenvalues are given by $y_{1}\left(1, \mu_{n}\right)$ and $y_{2}^{\prime}\left(1, \mu_{n}\right)$. By the Wronskian identity, they satisfy $y_{1}\left(1, \mu_{n}\right) y_{2}^{\prime}\left(1, \mu_{n}\right)=1$. By deforming the potential $q$ to the zero potential along the straight line $t q, 0 \leq t \leq 1$, one sees that $(-1)^{n} y_{1}\left(1, \mu_{n}\right)>0$. Hence the Floquet exponents are given by $\pm \kappa_{n}$ where

$$
\kappa_{n}:=-\log \left((-1)^{n} y_{1}\left(1, \mu_{n}\right)\right)
$$

and $\log$ denotes the principal branch of the logarithm. It turns out that together the $\mu_{n}$ 's and $\kappa_{n}$ 's form a system of canonical coordinates for $L_{0}^{2}$ (see [8]). The $\kappa_{n}^{\prime}$ 's also play an important role in proving the property of 1-smoothing of the periodic $\mathrm{KdV}$ equation [4. We want to determine the asymptotics of the $\kappa_{n}$ 's as $n \rightarrow \infty$. To state them, introduce for any $s \in \mathbb{R}_{\geq 0}$ the Sobolev spaces

$$
H_{0}^{s} \equiv H_{0}^{s}(\mathbb{T}, \mathbb{R})=H^{s}(\mathbb{T}, \mathbb{C}) \cap L_{0}^{2}(\mathbb{T}, \mathbb{R})
$$


and denote by $\langle\cdot, \cdot\rangle$ the standard inner product in $L_{0}^{2}(\mathbb{T}, \mathbb{R})$,

$$
\langle f, g\rangle=\int_{0}^{1} f(x) g(x) d x .
$$

In [3], the following theorem was proved.

Theorem $1.2([3])$. For any $N \in \mathbb{Z}_{\geq 0}$,

$$
\kappa_{n}=\frac{1}{2 \pi n}\left(\langle q, \sin 2 \pi n x\rangle+\frac{1}{n^{N+1}} \ell_{n}^{2}\right)
$$

uniformly on bounded subsets of $H_{0}^{N}$. Here $\ell_{n}^{2}$ denotes the $n^{\prime}$ th component of a sequence in $\ell^{2} \equiv \ell^{2}(\mathbb{N}, \mathbb{R})$.

Our aim is to extend Theorem 1.2 to any fractional order Sobolev space $H_{0}^{s}$ with $s \in \mathbb{R}_{\geq 0}$. To this end we need to extend various quantities to the complex Hilbert space $H_{0, \mathbb{C}}^{s}:=\left\{q \in H_{\mathbb{C}}^{s} \mid \int_{0}^{1} q(x) d x=0\right\}$. Let $L_{0, \mathbb{C}}^{2} \equiv H_{0, \mathbb{C}}^{0}$ and denote by $B_{0, \mathbb{C}}^{s}(R)$ the complex ball $B_{0, \mathbb{C}}^{s}(R):=\left\{q \in H_{0, \mathbb{C}}^{s} \mid\|q\|<R\right\}$. In Section 3 we prove the following

Theorem 1.3. For any $R>0$ there exists $n_{R}>0$ so that for any $n>n_{R}, \kappa_{n}$ can be extended analytically to $B_{0, \mathbb{C}}^{0}(R)$. Moreover, for any $N \in \mathbb{Z}_{\geq 0}$ the sequence $\left(\kappa_{n}\right)_{n>n_{R}}$ satisfies the estimate

$$
\kappa_{n}=\frac{1}{2 \pi n}\left(\langle q, \sin 2 n \pi x\rangle+\frac{1}{n^{N+1}} \ell_{n}^{2}\right)
$$

uniformly on $B_{0, \mathbb{C}}^{N}(R)$.

At the end of Section 3 we show that Theorem 1.3 allows us to apply Theorem 1.1 to generalize Theorem 1.2 to any fractional order Sobolev space.

Theorem 1.4. For any $s \in \mathbb{R}_{\geq 0}$

$$
\kappa_{n}=\frac{1}{2 \pi n}\left(\langle q, \sin 2 n \pi x\rangle+\frac{1}{n^{s+1}} \ell_{n}^{2}\right)
$$

uniformly on bounded subsets of $H_{0}^{s}$.

By the same method one can show that similar results hold for many other spectral quantities. We state without proof another such result. For $q \in L_{0}^{2}$, the periodic spectrum of $L(q)$ on the interval [0,2] is real and discrete. When listed in increasing order and with their multiplicities the eigenvalues satisfy

$$
\lambda_{0}<\lambda_{1}^{-} \leq \lambda_{1}^{+}<\lambda_{2}^{-} \leq \lambda_{2}^{+}<\ldots
$$

Using [3, Theorem 1.3, Theorem 1.4], one then shows with the help of Theorem 1.1 that the following result holds.

Theorem 1.5. For any $s \in \mathbb{R}_{\geq 0}$

$$
\left(\lambda_{n}^{+}+\lambda_{n}^{-}\right) / 2-\mu_{n}=\langle q, \cos 2 \pi n x\rangle+\frac{1}{n^{s+1}} \ell_{n}^{2}
$$

uniformly on bounded subsets of $H_{0}^{s}$. 
Related work. In the context of nonlinear PDEs, Tartar obtained a nonlinear interpolation theorem in [12] which later was slightly improved in [2, Theorem 1]. However, for nonlinear maps such as the ones encountered in the analysis of Schrödinger operators, the assumptions of these theorems are not satisfied and hence they cannot be applied. See also [7] and the references therein.

\section{Proof of Theorem 1.1}

The proof of Theorem 1.1 uses arguments of the proof of the Riesz-Thorin theorem on the interpolation of linear operators (see e.g. 1]). The key ingredient is Hadamard's three line theorem.

Proof of Theorem 1.1. As the case $p=1$ is similar we restrict our attention to the case when $1<p<\infty$. For any $k \in \mathbb{Z}$, denote by $F_{k}$ the $k$ 'th component of $F$. As $F: B_{\mathbb{C}}^{a}(R) \rightarrow \ell_{\mathbb{C}}^{p, \alpha+\beta b}$ is analytic, $F_{k}: B_{\mathbb{C}}^{a}(R) \rightarrow \mathbb{C}$ is analytic. In particular, the restriction $\left.F_{k}\right|_{B_{\mathbb{C}}^{s}(R)}$ of $F_{k}$ to $B_{\mathbb{C}}^{s}(R) \subseteq B_{\mathbb{C}}^{a}(R)$ is analytic for any $a \leq s \leq b$. Hence once the claimed bound for $\left.F\right|_{B_{\mathbb{C}}^{s}(R)}$ is established, the analyticity of $\left.F\right|_{B_{\mathbb{C}}^{s}(R)}: B_{\mathbb{C}}^{s}(R) \rightarrow \ell_{\mathbb{C}}^{p, \alpha+\beta s}$ will follow (cf. e.g. [5, Appendix A]). To prove the claimed bound consider $\varphi \in B_{\mathbb{C}}^{s}(R)$ and define the complex family

$$
\varphi_{z}(x):=\sum_{k \in \mathbb{Z}}\langle k\rangle^{s-z} \hat{\varphi}(k) e^{2 \pi i k x}
$$

where $z=u+i v \in \mathbb{C}, u, v \in \mathbb{R}$, and $\langle k\rangle:=1+|k|$. As $u$ and $v$ are real we see that for any $\varphi \in B_{\mathbb{C}}^{s}(R)$,

$$
\left\|\varphi_{u+i v}\right\|_{u}^{2}=\sum_{k \in \mathbb{Z}}\langle k\rangle^{2 u}\left|\langle k\rangle^{-u-i v+s} \hat{\varphi}(k)\right|^{2}=\sum_{k \in \mathbb{Z}}\langle k\rangle^{2 s}|\hat{\varphi}(k)|^{2}=\|\varphi\|_{s}^{2}<R^{2} .
$$

In particular, for any $v \in \mathbb{R}$,

$$
\left\|F\left(\varphi_{a+i v}\right)\right\|_{p, \alpha+\beta a} \leq M_{a} \text { and }\left\|F\left(\varphi_{b+i v}\right)\right\|_{p, \alpha+\beta b} \leq M_{b} .
$$

Similarly, for any $a \leq u \leq b$ and $v \in \mathbb{R}$,

$$
\left\|\varphi_{u+i v}\right\|_{a} \leq\left\|\varphi_{u+i v}\right\|_{u}=\|\varphi\|_{s}<R .
$$

Hence $\varphi_{u+i v} \in B_{\mathbb{C}}^{a}(R)$ for any $a \leq u \leq b$ and any $v \in \mathbb{R}$. In particular, $F\left(\varphi_{u+i v}\right) \in$ $\ell_{\mathbb{C}}^{p, \alpha+\beta a}$ and

$$
\left\|F\left(\varphi_{u+i v}\right)\right\|_{p, \alpha+\beta a} \leq M_{a} .
$$

As $\varphi_{s}=\varphi$ for any $\varphi \in B_{\mathbb{C}}^{s}(R)$, we need to prove the claimed estimate for $z=s$. Denote by $1<q<\infty$ the number conjugate to $p$, i.e. $\frac{1}{p}+\frac{1}{q}=1$, and consider an arbitrary sequence $\xi=\left(\xi_{k}\right)_{k \in \mathbb{Z}} \subseteq \mathbb{C}$ with finite support so that $\|\xi\|_{q,-(\alpha+\beta s)} \leq 1$. Similarly as in the case of $H_{\mathbb{C}}^{s}$, define for $z=u+i v \in \mathbb{C}, u, v \in \mathbb{R}$,

Then

$$
\xi_{z}:=\left(\langle k\rangle^{-\beta(s-z)} \xi_{k}\right)_{k \in \mathbb{Z}} .
$$

$$
\begin{aligned}
\left\|\xi_{u+i v}\right\|_{q,-(\alpha+\beta u)}^{q} & =\sum_{k \in \mathbb{Z}}\left(\langle k\rangle^{-(\alpha+\beta u)}\left|\langle k\rangle^{-\beta(s-z)} \xi_{k}\right|\right)^{q} \\
& \leq \sum_{k \in \mathbb{Z}}\left(\langle k\rangle^{-(\alpha+\beta s)}\left|\xi_{k}\right|\right)^{q} \leq 1
\end{aligned}
$$


Similarly as above, $\xi_{s}=\xi$, and for any $a \leq u \leq b$ and $v \in \mathbb{R}$,

$$
\left\|\xi_{u+i v}\right\|_{q,-(\alpha+\beta a)} \leq\|\xi\|_{q,-\alpha+\beta b} .
$$

Denote by $\langle\cdot, \cdot\rangle$ the $\ell^{2}$ dual pairing as well as its extension to $\ell_{\mathbb{C}}^{p, \alpha+\beta a} \times \ell_{\mathbb{C}}^{q,-(\alpha+\beta a)}$ and introduce the vertical strip

$$
S_{a, b}:=\{z=u+i v \mid v \in \mathbb{R}, a<u<b\}
$$

and its closure $\bar{S}_{a, b}$. To obtain the claimed estimate we want to apply Hadamard's three line theorem to the following function:

$$
f: \bar{S}_{a, b} \rightarrow \mathbb{C}, z \mapsto\left\langle F\left(\varphi_{z}\right), \xi_{z}\right\rangle
$$

where

$$
\left\langle F\left(\varphi_{z}\right), \xi_{z}\right\rangle=\sum_{k \in \mathbb{Z}} F_{k}\left(\varphi_{z}\right)\langle k\rangle^{-\beta(s-z)} \xi_{k} .
$$

As the support of $\xi$ is finite, the latter sum is finite, and hence the function $f$ is well defined and $\|\xi\|_{q,-\alpha+\beta b}<\infty$. In view of (2.2) and (2.4), $f: \bar{S}_{a, b} \rightarrow \mathbb{C}$ is bounded as for any $z \in \bar{S}_{a, b}$,

$$
|f(z)|=\left|\left\langle F\left(\varphi_{z}\right), \xi_{z}\right\rangle\right| \leq\left\|F\left(\varphi_{z}\right)\right\|_{p, \alpha+\beta a}\left\|\xi_{z}\right\|_{q,-(\alpha+\beta a)} \leq M_{a}\|\xi\|_{q,-\alpha+\beta b} .
$$

Note that on the strip $S_{a, b}$, the curves $z \mapsto \xi_{z} \in \ell_{\mathbb{C}}^{q,-(\alpha+\beta a)}$ and $z \mapsto \varphi_{z} \in B_{\mathbb{C}}^{a}(R) \subseteq$ $H_{\mathbb{C}}^{a}$ are analytic. As $F_{k}: B_{\mathbb{C}}^{a}(R) \rightarrow \mathbb{C}$ is analytic for any $k \in \mathbb{Z}$ it then follows that $f$, being a finite sum of analytic functions, is analytic on $S_{a, b}$ and continuous on the closure $\bar{S}_{a, b}$. Moreover, in view of (2.1) and (2.3), the following estimates hold for any $v \in \mathbb{R}$ :

$$
|f(a+i v)| \leq\left\|F\left(\varphi_{a+i v}\right)\right\|_{p, \alpha+\beta a}\left\|\xi_{a+i v}\right\|_{q,-(\alpha+\beta a)} \leq M_{a}
$$

and

$$
|f(b+i v)| \leq\left\|F\left(\varphi_{b+i v}\right)\right\|_{p, \alpha+\beta b}\left\|\xi_{b+i v}\right\|_{q,-(\alpha+\beta b)} \leq M_{b}
$$

Hence we can apply Hadamard's three line theorem to $f$ (cf. e.g. 9, Appendix to IX,.4]) to conclude that, for $s=(1-\lambda) a+\lambda b$ and any $v \in \mathbb{R}$

$$
|f(s+i v)| \leq M_{a}^{1-\lambda} M_{b}^{\lambda} .
$$

In particular, for $z=s$ one has

$$
|f(s)|=|\langle F(\varphi), \xi\rangle| \leq M_{a}^{1-\lambda} M_{b}^{\lambda}
$$

where we took into account that $\varphi_{s}=\varphi$ and $\xi_{s}=\xi$. The claimed estimate $\|F(\varphi)\|_{p, \alpha+\beta s} \leq M_{a}^{1-\lambda} M_{b}^{\lambda}$ then follows from the Hahn-Banach theorem and the fact that $\ell_{\mathbb{C}}^{q,-(\alpha+\beta s)}$ is the dual space of $\ell_{\mathbb{C}}^{p, \alpha+\beta s}$.

\section{Proofs of Theorem 1.3 and Theorem 1.4}

Before proving Theorem 1.3 we need to make some preparatory considerations. Note that for $q$ in $L_{0, \mathbb{C}}^{2}$ the operator $L(q)$ is no longer symmetric with respect to the $L^{2}$-inner product $\langle f, g\rangle=\int_{0}^{1} f(x) \overline{g(x)} d x$. The Dirichlet spectrum is still discrete, but the eigenvalues might be complex valued and multiple. We list them according to their algebraic multiplicities and in lexicographic ordering

$$
\mu_{1} \preccurlyeq \mu_{2} \preccurlyeq \mu_{3} \preccurlyeq \ldots
$$

where for any complex numbers $a, b$

$$
a \preccurlyeq b \text { iff }[\operatorname{Re} a<\operatorname{Re} b] \text { or }[\operatorname{Re} a=\operatorname{Re} b \text { and } \operatorname{Im} a \leq \operatorname{Im} b] .
$$


By [8] there exists for any $R>0$ an integer $m_{R}>0$ so that for any $q \in L_{0, \mathbb{C}}^{2}$ with $\|q\|:=\langle q, q\rangle^{1 / 2}<R$ the Dirichlet eigenvalues $\mu_{n} \equiv \mu_{n}(q), n \geq 1$, satisfy the following estimates:

$$
\left|\mu_{n}-n^{2} \pi^{2}\right|<\pi / 4 \quad \forall n>m_{R}
$$

and

$$
\left|\mu_{n}\right|<m_{R}^{2} \pi^{2}+\pi / 4 \quad \forall 1 \leq n \leq m_{R} .
$$

In particular, for any $n>m_{R}, \mu_{n}$ is a simple Dirichlet eigenvalue of $L(q)$ and hence analytic on the complex ball $B_{0, \mathbb{C}}^{0}(R)=\left\{q \in L_{0, \mathbb{C}}^{2} \mid\|q\|<R\right\}$. To see that $\kappa_{n}$ can be analytically extended to $B_{0, \mathbb{C}}^{0}(R)$ for $n$ sufficiently large we first note that by [8], the Floquet matrix $M(1, \lambda, q)$ is analytic on $\mathbb{C} \times L_{0, \mathbb{C}}^{2}$ and, with $\lambda=\nu^{2}, y_{1}\left(1, \nu^{2}, q\right)$ satisfies the following estimate:

$$
\left|y_{2}\left(1, \nu^{2}, q\right)-\cos \nu\right| \leq \frac{1}{|\nu|} \exp (|\operatorname{Im} \nu|+\|q\|) .
$$

As for $n>m_{R}$, one has $\left|\mu_{n}-n^{2} \pi^{2}\right|<\pi / 4$. It follows that $\nu_{n} \equiv \nu_{n}(q)=\sqrt[+]{\mu_{n}(q)}$ is well defined. Here $\sqrt[+]{z}$ denotes the principal branch of the square root. Then for any $n>m_{R}$ and $q \in B_{0, \mathbb{C}}^{0}(R), \nu_{n}=n \pi \sqrt[+]{1+z_{n}}$, where $z_{n}=\frac{\mu_{n}-n^{2} \pi^{2}}{n^{2} \pi^{2}}$ satisfies $\left|z_{n}\right| \leq \frac{1}{4 \pi n^{2}} \leq \frac{1}{10}$ and thus

$$
\left|\sqrt[+]{1+z_{n}}-1\right|=\left|\frac{\left(\sqrt[+]{1+z_{n}}-1\right)\left(\sqrt[+]{1+z_{n}}+1\right)}{\sqrt[+]{1+z_{n}}+1}\right| \leq\left|z_{n}\right|
$$

or

$$
\left|\nu_{n}-n \pi\right| \leq \frac{\pi}{4} \frac{1}{4 n} \leq \frac{1}{4 n} ; \quad\left|\operatorname{Im} \nu_{n}\right| \leq \frac{1}{4 n} .
$$

These estimates are used in the asymptotics of $y_{1}\left(1, \nu_{n}^{2}, q\right)$. As $(-1)^{n} \cos \nu_{n}=$ $1+\left(\nu_{n}-n \pi\right) \int_{0}^{1}-\sin \left(t\left(\nu_{n}-n \pi\right)\right) d t$ one concludes that $\left|(-1)^{n} \cos \nu_{n}-1\right| \leq \frac{1}{4 n}$ for any $n>m_{R}$ and as $\left|\nu_{n}\right| \geq n \pi-\frac{1}{4 n} \geq 2 n$ it follows from (3.1) that

$$
\left|(-1)^{n} y_{1}\left(1, \mu_{n}, q\right)-1\right| \leq \frac{1}{4 n}+\frac{1}{2 n} \exp \left(\frac{1}{4 n}+R\right)
$$

for any $n>m_{R}$ and $\|q\|<R$. Now choose $n_{R} \geq m_{R}$ so large that

$$
\frac{1}{4 n}+\frac{1}{2 n} \exp \left(\frac{1}{4 n}+R\right) \leq \frac{1}{2} \quad \forall n>n_{R}
$$

As a consequence, for any $q \in L_{0, \mathbb{C}}^{2}$ with $\|q\|<R$ and any $n>n_{R}$,

$$
\kappa_{n}=-\log \left((-1)^{n} y_{1}\left(1, \mu_{n}, q\right)\right)
$$

is well defined. We thus have proved.

Lemma 3.1. For any $R>0$ there exists $n_{R}>0$ so that $\forall n>n_{R}$

$$
\kappa_{n}: B_{0, \mathbb{C}}^{0}(R) \rightarrow \mathbb{C}, \quad q \mapsto-\log \left((-1)^{n} y_{1}\left(1, \mu_{n}, q\right)\right)
$$

is well defined and analytic.

Proof of Theorem 1.3. In view of Lemma 3.1 it remains to prove for any $R>0$, and any $N \in \mathbb{Z}_{\geq 0}$,

$$
\sum_{n>n_{R}} n^{2 N+2}\left|2 \pi n \kappa_{n}-\langle q, \sin 2 n \pi x\rangle\right|^{2}
$$


is bounded on $B_{0, \mathbb{C}}^{N}(R)$. Going through the arguments of the proof of Theorem 1.1 in [3], one sees that this is indeed the case.

It remains to show Theorem 1.4. As already mentioned in the introduction, we will use the result of Theorem 1.1 on nonlinear interpolation.

Proof of Theorem 1.4. By Theorem 1.2. Theorem 1.4 holds in the case where $s$ is an integer. Let $N \in \mathbb{Z}_{\geq 0}$ and $N<s<N+1$. For any given $R>0$ choose $n_{R}>0$ as in Theorem 1.3. It follows from Theorem 1.2 that there exists $M_{R}>0$ such that for any $q \in L_{0}^{2}$ with $\|q\|<R$ and any $1 \leq n \leq n_{R}$

$$
\left|2 \pi n \kappa_{n}-\langle q, \sin 2 \pi n x\rangle\right| \leq M_{R}
$$

and therefore

$$
\left(\sum_{n \leq n_{R}} n^{2(s+1)}\left|2 \pi n \kappa_{n}-\langle q, \sin 2 \pi n x\rangle\right|^{2}\right)^{1 / 2} \leq n_{R}^{s+3 / 2} M_{R}
$$

On the other hand, by Theorem [1.3, for any $n>n_{R}, \kappa_{n}$ extends analytically to $B_{0, \mathbb{C}}^{0}(R)$ and there exist constants $M_{N, R}, M_{N+1, R}$ so that

$$
\left(\sum_{n>n_{R}} n^{2(N+1)}\left|2 \pi n \kappa_{n}-\langle q, \sin 2 n \pi x\rangle\right|^{2}\right)^{1 / 2} \leq M_{N, R} \quad \forall q \in B_{0, \mathbb{C}}^{N}(R)
$$

as well as

$$
\left(\sum_{n>n_{R}} n^{2(N+2)}\left|2 \pi n \kappa_{n}-\langle q, \sin 2 n \pi x\rangle\right|^{2}\right)^{1 / 2} \leq M_{N+1, R} \quad \forall q \in B_{0, \mathbb{C}}^{N+1}(R) .
$$

Hence Theorem 1.1 applies and implies that with $0 \leq \lambda \leq 1$ chosen so that $s=$ $(1-\lambda) N+\lambda(N+1)$,

$$
\left(\sum_{n>n_{R}} n^{2(s+1)}\left|2 \pi n \kappa_{n}-\langle q, \sin 2 n \pi x\rangle\right|^{2}\right)^{1 / 2} \leq M_{s, R} \quad \forall q \in B_{0, \mathbb{C}}^{s}(R)
$$

where $M_{s, R}=\left(M_{N, R}\right)^{(1-\lambda)}\left(M_{N+1, R}\right)^{\lambda}$. From this together with (3.2), the claimed result follows.

\section{ACKNOWLEDGMENT}

The motivation of this paper originated from our observation that in the proof of Theorem 6.1 in the paper [10] of A. Savchuk and A. Shkalikov, the theorem of Tartar on nonlinear interpolation [12] cannot be applied as stated by the authors. After having pointed this out to A. Shkalikov, he sent us the statement of an unpublished interpolation theorem obtained by them earlier, which can be used to prove their results. Due to unfortunate personal circumstances their paper with a proof of this interpolation theorem was delayed. In order to be able to further progress on our projects, we came up with our own version in the set-up needed for our applications and with an optimal estimate on the bounds of the interpolating maps. After having sent our preprint to A. Shkalikov, he sent us a preprint with the proof of their theorem [11. As the two papers turned out to be quite different, we agreed to publish them independently. 


\section{REFERENCES}

[1] Jöran Bergh and Jörgen Löfström, Interpolation spaces. An introduction, Springer-Verlag, Berlin, 1976. Grundlehren der Mathematischen Wissenschaften, No. 223. MR0482275 (58 \#2349)

[2] Jerry Bona and Ridgway Scott, Solutions of the Korteweg-de Vries equation in fractional order Sobolev spaces, Duke Math. J. 43 (1976), no. 1, 87-99. MR0393887 (52 \#14694)

[3] T. Kappeler, B. Schaad, and P. Topalov, Asymptotics of spectral quantities of Schrödinger operators, Spectral geometry, Proc. Sympos. Pure Math., vol. 84, Amer. Math. Soc., Providence, RI, 2012, pp. 243-284, DOI 10.1090/pspum/084/1360. MR2985321

[4] T. Kappeler, B. Schaad, P. Topalov: Qualitative features of periodic solutions of $K d V$, arXiv:1110.0455, to appear in Comm. Partial Differential Equations

[5] Thomas Kappeler and Jürgen Pöschel, $K d V$ \& $K A M$, Ergebnisse der Mathematik und ihrer Grenzgebiete. 3. Folge. A Series of Modern Surveys in Mathematics [Results in Mathematics and Related Areas. 3rd Series. A Series of Modern Surveys in Mathematics], vol. 45, SpringerVerlag, Berlin, 2003. MR1997070 (2004g:37099)

[6] S. B. Kuksin, Oscillations in space-periodic nonlinear Schrödinger equations, Geom. Funct. Anal. 7 (1997), no. 2, 338-363, DOI 10.1007/PL00001622. MR1445390 (98e:35157)

[7] Lech Maligranda, Lars Erik Persson, and John Wyller, Interpolation and partial differential equations, J. Math. Phys. 35 (1994), no. 9, 5035-5046, DOI 10.1063/1.530829. MR.1290918 (95g:46065)

[8] Jürgen Pöschel and Eugene Trubowitz, Inverse spectral theory, Pure and Applied Mathematics, vol. 130, Academic Press Inc., Boston, MA, 1987. MR894477 (89b:34061)

[9] M. Reed, B. Simon: Methods of modern mathematical physics, Vol. 2, Academic Press, Boston, 1975

[10] A. M. Savchuk and A. A. Shkalikov, On the eigenvalues of the Sturm-Liouville operator with potentials in Sobolev spaces (Russian, with Russian summary), Mat. Zametki 80 (2006), no. 6, 864-884, DOI 10.1007/s11006-006-0204-6; English transl., Math. Notes 80 (2006), no. 5-6, 814-832. MR2311614 (2008d:34160)

[11] A. Savchuk, A. Shkalikov: On the interpolation of analytic maps, arXiv:1307.0623.

[12] L. Tartar, Interpolation non linéaire et régularité (French), J. Functional Analysis 9 (1972), 469-489. MR0310619 (46 \#9717)

Mathematics Institute, University of Zurich, Winterthurerstr. 190, CH-8057 Zurich, SwitzerLAND

Department of Mathematics, Northeastern University, 360 Huntington Avenue, Boston, Massachusetts 02115 